uses their vital strength and rids the system of poison just about as much, in proportion, as bleeding did in the olden days.

You encourage a general peritonitis by peristaltic whipping of the bowels, whereas if they are permitted to lie quiet the infection may localize. Our practice is to splint the bowel by the free use of morphin and an ice cap upon the abdomen and by using soapsuds enemas for daily evacuation of the bowels. If the abscess localizes in the cul de sac, drain through the vagina.

Since this practice our mortality has been greatly reduced.

Dr. W. H. Vogt, St. Louis, Io.-1 was very glad to hear Dr. Altman take a conservative stand in the treatment of puerperal sepsis. The too active treatment of these cases usually does more harm than good. The routine curettage of such cases is generally adnitted to be poor treatment.

Regarding the administration of the antistreptococcic serums, I have only this to say: that I have never seen any definite benefit derived from them, though I use them in some individual cases. When used they must be in very large doses if one hopes for any good results at all.

No method of treatment seems to gain universal results and I believe that those cases that recover have been of a milder type. I am satisfied that if we find the streptococcus in the blood stream the case is hopeless to begin with and that any method of treatment would be of no avail. On the other hand, if this is not the case and we have a mild form of infection, Nature perhaps takes care of it without our assistance, except that we may aid the patient in fighting such infection.

Dr. W. A. Fowler, Oklahoma City, Okla.-Dr. Altman rightly lays greatest enphasis upon the danger of exploring and treating the uterine cavity in puerperal fever. It should be resorted to only in cases in which sapremia is clearly indicated and in which there is a discharge that is either very free and foul-smelling or very bloody. All authorities seem to be agreed that if this procedure is resorted to the gentlest possible means should be used and by no means should there be a repetition.

As to Dr. Altman's suggestion that the patient be given ergot in the beginning of this condition, I believe it unwise. The most important factor in the treatment of sepsis is rest, and rest particularly of the infected organ. Ergot will only undo Nature's effort in this direction. When we have the signs of peritonitis the treatment should consist of the Foxler position, nothing at all by mouth, the Murphy drip and morphin for relaxation. This routine will splint the bowels and give Nature a chance to wall off the infection.

Dr. Altman (closing).-If my paper should help to cut down the mortality of this deadly disease, my object will have been attained. I make no claim for anything new.

\section{TREATMENT OF FRACTURES NEAR THE SHOULDER, ELBOW AND WRIST*}

\author{
BY W. W. HARPER, M.D., \\ Selma, Ala.
}

In treating fractures near the large joints, one must bear in mind not only the attainment of a good union of the fracture, but the maintenance of perfect joint function. While the joints of the lower extremities are similar anatomically to those of the upper, their function is somewhat different. The joints of the lower extremities are made to bear weight and for locomotion, while the upper have as their purpose complicated movements. It is, therefore, evident that a good functional result in a lower extremity joint might be considered a poor one in an upper.

\section{FRACTURE OF HUMERUS}

All fractures of the surgical neck of the humerus should be treated in abduction to overcome the pull of the adductors and to relax the deltoid. The position of abduction is maintained by means of a wooden tripod and plaster of Paris. The arm rests upon one leg of the tripod, while the other leg rests against the side of the chest. The tripod is held to the side by adhesive. The upper extremity, shoulder and chest, are now encased in plaster. This immobilizes the fracture and secures union with good alignment and a minimum of callus.

\section{FRACTURE OF THE ELBOW}

The elbow is a hinge joint and its function is to allow flexion and extension. With one exception (fracture of the olecranon) this principle is laid lown: all fractures of the elbow must be treated in acute flexion. The position of flexion is maintained by bandaging the forearm to the arm with a four-inch adhesive. Care must be exercised to provide for swelling at the elbow. The wrist is to be well padded with cotton and then swung from the neck by a bandage.

* Read before the Southern States Association of Railway Surgeons, Auxiliary of the Southern Medical Association, Fifteenth Annual Meeting, Hot Springs, Ark, Nov. 14-17, 1921. 
The after-treatment of these fractures is most important, and for its perfection we are indebted to $\mathrm{Sir}$ Robert Jones. At the end of ten days he cuts the adhesive and lets the forearm down ten degrees. He then fixes the elbow in this position, provided the patient can work his forearm in that arc without pain. If not, he returns to the first position for another ten clays. Then he drops down ten degrees every ten days until within ten or fifteen degrees of normal, when the bandage is removed and the patient is allowed to use his elbow. If, after two months of use, there is not full extension, the remaining adhesions are broken under an anesthetic.

There is one fracture at the elbow joint that frequently calls for an immediate open operation, and that is a fracture of the lateral condyle. In some fractures of the lateral condyle the broken fragment rotates on itself and becomes interposed between the head of the radius and the humerus. External manipulation usually fails to reduce this dislocation. The only thing to do is an cpen operation to pry the bone back into place; for if the fragment is improperly reduced, the patient is crippled for life. In this operation it is not necessary to open the elbow joint, as the fracture is just below the skin.

\section{COLLES' FRACTURE}

There are a number of doctors who are not familiar with Jones' method of reducing fractures near the lower end of the radius. All should know his method, for by it one not only can at the time do a perfect reduction, but reduction can be effected at the end of a month in unrecognized cases.

$\mathrm{Mr}$. Jones makes this assertion: "A man who walks the floor the first night after his reduction has supposedly been made, has not had that reduction made." In other words, if the reduction has been made the patient will be comfortable. Of course, all patients should be anesthetized.

I recently saw a woman who was supposed to have a dislocation. It was treated as a dislocation. She suffered all the time and came to me at the end of a month. A Colles' was diagnosed. I gave an anesthetic, and with my own hands reduced this onemonth-old fracture, applied plaster of Paris splints, leaving out fingers, and told her to go ahead with her work. She was a nurse and continued to nurse the children of the household as she had before the accident. After the reduction there was no further pain and there was a perfect functional result. Having effected a reluction, $\mathrm{my}$ rule is to put the forearm in palma: llexion for ten days, then straight for ten days. and for the next ten days in slight dorsi-flexion. Use moulded plaster splints down to the metacarpo-phalangeal joints. Encourage the patient to use the hand the next day. This treatment has given the writer the best functional results.

In fractures of the wrist that are liable to end in ankylosis, the wrist must be treated in dorsi-flexion, as this will give the patient a good grasp if ankylosis does occur.

In the stiff wrists, due to extra-articular adhesions, do not give an anesthetic and vigorously manipulate the joint. The result will be more and firmer adhesions. It is better to do all you can without a general anesthetic, urging the patient to bear all he can. Instead of making many up and down movements, make one continuous pull, stretehing everything. Then when the patient can bear no further pull, stop until the next day. Repeat this every day until the result is obtained.

\section{DISCUSSION}

Dr. Edtcard T. Nevell, Chattamonga, Tenu.Dr. Harper speaks of using a triangular splint. That is one $I$ have used for a number of years with a great deal of success, with a slight difference in the lengths. In applying this splint I usually use broad adhesive plaster and strap the splint to the arm, then to the body, all the way roand. In going around with the plaster of Faris, with the splint in that position, I d not put the plaster around the splint. I put it nore around the arm and over it, around the bod: in front and in the back. With two pieces of thick flannel attached to it one can pull it up and get more of a shoulder brace. The splint and packing that Dr. Harper has suggested, while it is quite old, is very practical and gives the best results in this condition. It allows a certain amount of motion, and as we all know, if we have a little motion we get better results and better union than where we have complete immobilization. It is for that reason that we get such splendid results here.

The treatment I have been accustomed to use in the elbow is the anterior and posterior molded splint. The Doctor uses a plaster bandage or possibly adhesive plaster. I use the molded splint and a flexion about at right angles. It is 
very important that we should have the arm flexed at least to right angles, because if we do get stiffness, which we are liable to have in an injury of this joint or any other joint, we have the arm in a position where it can be used. The patient can dress his or her hair, can wash the face, and can eat.

We also know that there is a great tendency to draw the arm downward; that the posterior muscles are more powerful, and the tendency is to have an arm in extension. If it is in partial extension it is of very little functional value. So if we put the arm up in flexion and keep it there, if we get a stiff joint or partially stiff joint, we have quite a serviceable arm.

In regard to placing the patient under anesthesia for breaking up adhesions, ete., I believe the Doctor meant to stress the point particularly that he was not very much in favor of foreibly breaking up adhesions and callus formations, because they frequently form again. It would be much better to resort to hydrotherapy and massage under a competent person. It is nuch easier to get motion if you have the arm up and work it down than it is if you have it down and try to work it up. So if you have it up let it down only about ten degrees, as the Doctor suggested, and if it works well there, and good motion is obtained, you can continue to increase the angle from time to time.

There are, of course, many ways of setting Colles' fractures. There is the Jones method. But the old method which I have used is the "shakehands" method. The patient is under anesthesia. Usually the distal fragments are posterior and the proximal underneath. First, increase the fracture. Then as you come to the fracture line, slide down with the thumb the posterior displaced fragment. I find it to be better than the Jones method. Take hold of the right hand with your right hand or the left with your left, and grasp the other arm with your hand, using your knee as a fulcrum.

Using the fluoroscope, we stand a better chance of getting good results. I do not mean that the fuoroscope is 100 per cent perfect, but it is of great assistance. Where possible, all fractures should be $x$-rayed before setting them, fuoroscoped when you set them, and $x$-rayed after the setting. Possibly other radiograms should be made later to note the progress. The fluoroscope will give great assistance in setting. One can look at the fracture and sce if there is good approximation of the fragments. Even though it be not perfect, one may be satisfied that with the approximation and with the alignment obtained there will be a good enough result. Often there is a fairly good approximation, but we go on trying to do better and end by doing worse. The fuoroscopic examination at the time is of great value.

Dr. T. H. Hancock, Atlanta, Ga.-The Jones method of reducing a Colles' fracture can possibly be used by Dr. Harper. He has short fingers and a powerful grip. I am the strongest man around my district, but I cannot reduce these fractures by the Jones method. The wrist must be pit in cxtreme cxtension before the reduction can be made.

Prof. L. S. Pileher was the one who first described the reduction of this fracture. He says you must bring the hand and wrist back and nake the traction with it in extreme extension. You do not have to break it worse.

Dr. W. S. Audcrson, Memphis, Teun.-In fractures of the elbow joint it has been my practice to put the $\mathrm{arm}$ in acute flexion, that is, in the Jones position. I do that for two reasons. If perhaps we should get an ankylosis there in the acute flexion, the patient will have more use of the arm and can feed himself. If we get an ankylosis with the arm in probably the right angle position, it is very difficult to use the arm to any advantage. By putting the arm in the acute flexion we get relaxation of all the ligaments, and thus the fractures are easily kept in position.

In Colles' fracture the secret of success is proper reduction at the time. After this reduction has been obtained any type of splint can maintain it there. If that is done, then the secret of proper joint flexion is manipulation of the joint. The secret of all fractures is, if possible. to get an early passive motion of the joint and thus a flexible joint.

Dr. Isidore Cohn, Ncw Orleums, La.-In referring to the triangle, Dr. Harper has described part of the treatment suggested in a paper read before the Section on Surgery of the Southern Iredical Association in 1913 and afterward pub. lished in the JourNat. Dr. Harper did not stress sufficiently the importance of maintaining external rotation. The deltoid abducts the elbow contraction of the biceps and triceps, actually shortens the arm and the proximal end of the distal fragment, that is, of the shaft, is brought closer into the chest by the action of the pectoralis major and latissimus dorsi. The pectoralis major, in contracting, has a tendency to adduct the arm. That being favored by the abduction of the elbow by the deltoid, allows not only the adduction of the shaft but the internal rotation as a result of the contraction of the latissimus dorsi. In order to maintain abduction and external rotation, it is necessary to immobilize the elbow as well as the shoulder. Immobilization of the elbow not only maintains abduction and external rotation, but it avoids and overcomes the action of the biceps and triceps which have a tendency to produce an overriding deformity.

In order to prevent the triangle from slidin: hackward, as it may do, we bring our plaster through the one angle of the triangle and instead of going forward with it, simply bring it back in the opposite direction. That prevents the triangle from going forward. The inner side of that triangle should extend down to the iliac crest. We should have as much abduction as we possibly can. It is important to remember about getting external reduction.

Recently I have reviewed the experience with elbow fractures in our Clinic for the last ten years. It is important to know that you cannot reduce these just by hyperflexion. To reduce the deformity, hyperextend the elbow and make trac- 
tion and countertraction. This will enable you to increase the distance between the shaft and the lower fragment and then hyperflexion is easy enough.

In fractures of the lateral condyles, supination is the position, because from the lateral side of the shaft of the humerus and the lateral side of the lower end of the humerus we have attached our supinator longus, particularly. If we pronate the arm we have a deformity, because the muscles which originate from the medial condyle are contracting and pulling the lateral condyle away from its place. Therefore, in fractures of the iateral condyle complete supination is the position. In fractures of the medial condyle, on the other hand, if we have complete supination, the supinators contract more and this has a tendency to aid the pronator to pull the medial condyle away from the shaft.

It is important to remember that in the wrist you do not need to wait until ten days afterward to find out that the patient is going to have complete functional results. E. H. Skinner, of Kansas City, describes a very efficient method. We have planes passing through the middle of the long axis of the shaft of the radius and at right ancles to this a perpendicular is dropped from the tip of the ulna styloid. SIore than half of the base of the radius should be below the intersection. Under those conditions, if we have that in the picture, we can be well satisfied that unless the patient is immobilized too lonb, we shall have good results.

Dr. T. E. Abernathy, Chuttanooga, Tenu--In a fracture of the shoulder where the head of the bone is broken off and rolls, which it does as a rule toward the outer aspect of the shoulder, it is necessary to have extension and counter-exten sion to get it into place. The triangular splint is all right in many cases, especially if the bone is broken below the surrical neck in the shaft. But where the head of the bone rotates at the fracture, the only way that it can be kept in place is by extension and counter-extension such as you would put on the femur. We had a case not long ago of that character, and first put it up with the old-fashioned, wedge-shaped splint. which somewhat resembles the triangular splint. We $x$-rayed it. It would not stay in place. We tried different methods. Finally we put the patient to bed on her back, took a plank about a foot wide and laid the arm out that way with considerable extension and left it there two weeks. We $x$-rayed it in the meantime. At the end of two weeks the bones were in perfect apposition, the arm being straight out. We left it there for about a week, put it in plaster of Paris in that position and left it there for a month. We then took it down. After two months she had good use of the shoulder. There is no ankylosis and there is almost perfect function of the joint. I believe that is the best way to correct fractures where the head of the bone is broken oft.

Dr. W. H. Blake, Shefficld, Ala.-There is a fracture just above the elbow that does not include the joint. We have very little trouble under the fluoroscope, possibly, in reducing these fractures, but we do have trouble in holding them in position until our plaster splint hardens. I have a piece of five-eighths iron rod crooked. It comes down several inches below the elbow and roes several inches above the shoulder. I flex the arm at right angles and place plenty of padding just in front of the elbow. Over that I put with plenty of padding an ordinary piece of tin, which is bent to fit the arm. The tin is imbedded in the dressing and it must be comfortable after the dressing is applied. Then with a loop of gauze passing beneath the axilla and fastened to the end of the iron rod, which is entirely out of the way and another piece passing over the carved piece of tin and attached to the loop at the lower end of the bent rod, which is extended by a screw, under the fluoroseope I turn the screw until I get the fracture in apposition. Then I place on plaster, fitting it snugly under axilla and over the tin in front of the elbow. The extension and counter-extension which holds the fracture in place must be against and under the arm. The whole device is to overcome the dificulty we have in holding the fracture in apposition while the plaster hardens. The whole renson for the tin is to avoid discomfort by distribut ing the pressure in front of the elbow. I pad it thoroughly and make the dressings as loose as I can to hold the fractured bone in position.

Dr. Durean Evc, Nashville, Tenn.-I have nothing to suggest. The plan I always use is levis' formula for reduction. There are three well known formulas: the one that Dr. Harper uses, the Murphy method, and the Levis plan. Pilcher did show that in a fracture of the lower end of the radius there was a portion of the dorsal periosteum that acted as a binding band and held the fragments in deformity, but Dr. Levis proposed to relax this dorsal periosteum in order to reduce the fracture.

I agree with Dr. Harper that no fracture, especially of or near a joint, should be reduced except under anesthesia.

The MIurphy plan requires much strength in one's thumbs or fingers, while the plan of Levis is readily performed in three motions. First, make dorsal flexion carrying the hand well back upon the forearm (hyper-flexion) to relax the dorsal periosteum. Then traction is made to separate the fragments and finally force flexion to engage the fragments in reduction. You can always determine whether a Colles' fracture is reduced by finding out if the patient can flex his hand normally. Any plan of immobilization will answer if you begin passive movements early. You can use an ordinary shingle. You can use, as I frequently do, the Bond splint, which gives semi-flexion of the wrist and semiextension of the fingers, making the patient very comfortable.

I make my patients, particularly if they are beyond fifty years of age, use hot water and play ball, as I call it. I have them get an ordinary soft rubber ball and amuse themselves by almost continuously moving the fingers. This prevents ankylosis. There will be no deformity in $a$ Colles' fracture if it is properly reduced. 